\title{
Eliminating HIV transmission through breast milk from women taking antiretroviral drugs
}

\author{
Ameena Goga and colleagues argue that frequent testing of maternal viral load is needed to \\ eliminate HIV transmission through breast milk in low and middle income settings
}

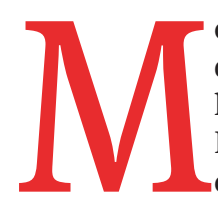

others taking antiretroviral drugs with low plasma viral loads may still transmit HIV to their breastfeeding children. Given the widely acknowledged benefits of breastfeeding, eliminating the risk of vertical transmission of HIV through breast milk must be a priority.

Transmission from mother to child via breast milk is influenced by maternal HIV viral load and occurs through cell-free and cell associated HIV-1. ${ }^{1}$ In women living with HIV, breast milk contains quiescent CD4 cells with high capacity to produce HIV and activated CD4 cells with replicating HIV, despite the presence of suppressive antiretroviral treatment. ${ }^{1}$ Additionally, transfer of maternal cells through breastfeeding induced microchimerism may establish permanent HIV reservoirs in breastfeeding infants. $^{2}$

Nonetheless, breast milk has multiple immediate and long term advantages:

\section{KEY MESSAGES}

- Mothers taking antiretroviral drugs with low detectable plasma viral loads may still transmit HIV.

- This is a result of challenges with antiretroviral treatment adherence and retention in care, viral load rebound between the viral load test intervals, transmission from a breast milk cellular reservoir, or postpartum or peripartum viral rebound.

- Point-of-care technologies for viral load testing during pregnancy and breastfeeding must be scaled up and results acted on to reduce transmission through breast milk.

- The utility of additional complementary interventions such as long acting maternal antiretroviral drugs, extended prophylaxis, broadly neutralising antibodies, or vaccines in infants whose mothers are receiving antiretroviral treatment needs further investigation. it contains bacterial genes facilitating carbohydrate, amino acid, and energy metabolism ${ }^{3}$; contributes to almost $40 \%$ of the infant microbiome during the first 30 days of life ${ }^{3}$; modulates the human virome ${ }^{4}$; reduces the risks of communicable and non-communicable diseases in childhood and adulthood ${ }^{5}$; and is associated with better IQ, educational attainment, and income at age 30 years. ${ }^{6}$ Consequently, there is an urgent need to eliminate transmission of HIV through breast milk.

Although HIV and infant feeding guidelines previously recommended avoiding breastfeeding or reducing duration, ${ }^{7-9}$ by 2016 modelling showed that in a setting where the mortality risk associated with avoiding breastfeeding is high, then 24 months of breastfeeding and maternal triple antiretroviral treatment maximises child HIV-free survival. ${ }^{10}$

Maternal antiretroviral treatment or infant prophylaxis during breastfeeding reduces the risk of HIV transmission through breast milk. ${ }^{11-14}$ Since 2016, all Global Plan priority countries (where 90\% of the world's pregnant women with HIV live) recommend lifelong antiretroviral treatment for pregnant and lactating women with HIV. ${ }^{15} 16$ Additionally, in 2016 the World Health Organization recommended that women taking antiretroviral drugs should breastfeed for at least 12 months and may continue breastfeeding for up to 24 months or longer (similar to the general population) while being fully supported for adherence to antiretroviral treatment. ${ }^{17}$

WHO guidelines for HIV plasma viral load monitoring are not specific to pregnancy and lactation: a plasma viral load test is recommended at six months and 12 months, and then every 12 months. ${ }^{18}$ Breast milk HIV viral load may be a better predictor of breast milk transmission than plasma viral load ${ }^{1}$; however, routine monitoring of breast milk viral load is costly, not widely performed, and not practical.

We have previously highlighted the increasing contribution of breast milk transmission to overall mother-to-child transmission in low and middle income countries because of challenges with early initiation of antiretroviral treatment and postnatal treatment adherence. ${ }^{19} \mathrm{We}$ have also highlighted the contribution of maternal prevalent or incident HIV infections to the paediatric HIV case rate ${ }^{16}$ 1920 and proposed that current strategies may fall short of eliminating breast milk transmission. ${ }^{19}$ Complementary interventions such as broadly neutralising antibodies or vaccines are needed. ${ }^{19} \mathrm{We}$ have also emphasised the critical need to dismantle structural barriers ${ }^{16}$ and strengthen routine cohort monitoring of women living with HIV and their children to determine long term outcomes. ${ }^{21}$ Here we consider whether we can eliminate breast milk transmission of HIV in women taking antiretroviral drugs.

\section{Risk of transmission through breast milk during treatment}

The risk of transmitting HIV through breast milk is not zero if women with HIV are not consistently and completely suppressed at $<50$ copies/mL plasma viral load throughout breastfeeding (see supplementary table 1 ; bmj.com). A major concern is that HIV transmission via breast milk has been described in women with previously documented viral load suppression; these findings may be because viral load measurements are taken or reviewed infrequently during breastfeeding. ${ }^{22-25}$

In many countries it is assumed that a viral load of $<1000$ copies/mL is associated with a low risk of mother to child transmission; however, in a South African study, women with a viral load of $<1000$ copies/mL at delivery accounted for $43 \%$ of early mother to child transmission (median age 44 days, interquartile range (IQR) 42-49 days). ${ }^{26}$ If we extrapolate from a meta-analysis that included women taking antiretroviral drugs (with detectable or undetectable HIV viral load) and assume a $20 \%$ maternal HIV prevalence, six month mother to child transmission among women taking antiretroviral drugs 
translates into 220 new infections per 100 000 live births, almost four times higher than the mother to child transmission elimination target of $\leq 50$ new infections per 100000 live births. ${ }^{27}$ In this context, 12 month mother to child transmission translates into 580 new paediatric HIV infections per 100000 live births-more than 10 times the global target. ${ }^{27} 28$ In a study in Malawi (October 2014 to May 2016), women taking antiretroviral drugs with low detectable viral load (defined as $\geq 40-1000$ copies/mL) contributed an excess of 460 new infant HIV infections annually, which translates into 60 additional infections per 100000 births annually. ${ }^{29}$

If breast milk mother-to-child transmission occurs despite maternal antiretroviral treatment, as a result of prolonged or intermittent maternal viraemia independent of health system and individual barriers to viral suppression, then the utility of additional or complementary interventions needs investigating. Potential interventions include long acting antiretrovirals or intermittently administered broadly neutralising antibodies or vaccines that could induce passive or active immunity without reliance on daily administration. Promisingly, in neonatal macaque models, combined administration of broadly neutralising antibodies PGT121 and VRC07-523 administered within 30 to 48 hours of oral simian HIV exposure mediated effective post-exposure prophylaxis in infant macaques. ${ }^{3031}$

\section{Risk factors for breast milk transmission}

Both maternal breast milk viral load and plasma viral load are predictors of breast milk HIV transmission. ${ }^{23}$ When single dose nevirapine for mother and baby was the main intervention to prevent transmission, maternal plasma viral load $>50000$ copies/ $\mathrm{mL}$ accounted for $37 \%$ (95\% confidence interval $22 \%$ to $51 \%$ ) of breast milk transmission between 6 weeks and 12 months. ${ }^{32}$ Multivariable analyses of data gathered in Malawi between 2014 and 2016 showed that maternal viral load was the only important predictor of transmission. ${ }^{29}$ Consequently, frequent maternal plasma viral load monitoring and management for all pregnant and breastfeeding women are critical to achieve and maintain plasma viral load below the detection threshold.

\section{Maternal plasma viral load monitoring}

Despite increasing access to lifelong antiretroviral treatment, the coverage of plasma viral load monitoring during breast- feeding ranges from 38\% to $98 \%$ in Global Plan priority countries. ${ }^{2933}$ Non-pregnant adult guidelines recommend six monthly viral load monitoring, but this may result in only $31 \%$ viral load testing coverage during pregnancy $^{34}$; reasons for this include late antenatal booking, limited number of antenatal visits, underuse of routine maternal viral load monitoring, stigma, and inoperability of machines to measure viral load. ${ }^{35} 36$

Since 2019, some low and middle income countries, such as South Africa, recommend viral load testing at antenatal care booking (or three months after antenatal antiretroviral treatment initiation or switching), at delivery, and then six monthly. ${ }^{37}$ If viral load is $\geq 1000$ copies/mL or 500-<999 copies/mL then counselling and repeat viral load testing are recommended 4-6 weeks or 8-10 weeks later, respectively. Infant antiretroviral prophylaxis is extended beyond six weeks or includes two drugs only if maternal viral load is $\geq 1000$ copies $/ \mathrm{mL}$. However, mother-to-child transmission can occur when viral load is $<1000$ copies/mL. ${ }^{37}$ Consequently, current recommendations would not eliminate the risk of breast milk transmission.

Regardless of setting or year, the prevalence of plasma viral load suppression among women with HIV taking antiretroviral drugs is suboptimal (see supplementary table 2; bmj.com). In rural Uganda, by 2015 five year retention in care was 90\% among women starting antiretroviral treatment during pregnancy, decreasing to $67.5 \%$ if those not followed up were assumed to be out of care. ${ }^{38}$ Among women with HIV retained in care, viral suppression ( $\leq 400$ copies $/ \mathrm{mL}$ ) was $89.6 \%$ (95\% confidence interval $83.2 \%$ to $94.2 \%$ ), falling to $80.7 \%$ ( $73.4 \%$ to $86.7 \%$ ) among participants enrolled in the study, and to $60.5 \%$ (53.6\% to $67.3 \%$ ), assuming that those not enrolled in the study were not suppressed. ${ }^{38}$

\section{Scaling up viral load monitoring}

Breastfeeding women are a priority population for repeated plasma viral load measurements. However, in many Global Plan priority countries viral load monitoring scale-up is limited because of logistical challenges relating to plasma specimen collection. This requires EDTA tubes, which are often unavailable, can break in transit, and require storage, a cold chain, timely centrifugation, and transportation to central laboratories. ${ }^{39}$

Some countries have used dried blood spot or plasma separation cards to scale up viral load testing; however, dried blood spot viral load testing cannot reliably measure viral loads $<1000$ copies/mL. ${ }^{39}$ Furthermore, the median time between sample collection and availability of results can be as long as 72 days. ${ }^{4041}$ Investing in mobile health technologies for use by clinicians and patients may improve turnaround times and prompt action for high viral load measurements. Plasma separation cards have a slightly lower limit of detection (790.2 copies/mL (95\% confidence interval 658.9 to 1003.6 copies/ $\mathrm{mL})),{ }^{41}$ are cost neutral, ${ }^{39}$ and are easier to use.

Point-of-care viral load testing is a promising technology as results are available about two hours after sample collection. An open label, non-inferiority, randomised controlled trial found that point-of-care viral load monitoring with task shifting significantly improved retention and viral suppression among HIV infected adults in South Africa. ${ }^{42}$ However, some point-of-care technologies cannot detect viral loads of $<50$ copies/ $\mathrm{mL}$. A systematic review for the Cepheid GeneXpert reported a pooled sensitivity of $96.5 \%$ (95\% confidence interval 95.1 to 97.5$)$ and pooled specificity of $96.6 \%$ (92.9 to 98.4) for a treatment failure threshold of 1000 copies/mL; two publications on the Abbott m-PIMA device sensitivities of $95.4 \%$ (89.7 to 98.5$)$ and $97.1 \%$ (94.2 to 98.8$)$ and specificities of $96.0 \%$ (93.7 to 97.6 ) and $76.9 \%$ (69.8 to 83.1) for a treatment failure threshold of 1000 copies $/ \mathrm{mL}^{43}$

Since March 2021 WHO has recommended point-of-care viral load testing to monitor treatment in people living with HIV. ${ }^{43}$ Consequently, scaling up point-of-care viral load testing urgently requires implementation and integration into routine care, including regulatory approval, training, monitoring, planning for supply chain, reagent forecasting, human resources, device maintenance, quality assurance, and factoring in an additional two hour wait by patients. ${ }^{43} 44$ Moreover, technological innovations to detect viral loads of $<50$ copies/mL are needed. ${ }^{45}$

\section{Other challenges}

The prospect of viral load suppression exists only if mothers access antiretroviral treatment. Historically, Global Plan priority countries have fragile health systems. For example, in sub-Saharan Africa, $83 \%$ of women have at least one antenatal visit-mainly during the second or third 
trimester-but only 55\% have at least four antenatal visits. ${ }^{46}$ Additionally, a metaanalysis of 1703 antiretroviral treatment clinics in 35 countries (2010-14) showed that $37.5 \%$ had at least one antiretroviral treatment out of stock during a 12 month period. ${ }^{47}$ Similarly, in South Africa, in $2015,20 \%$ of 2370 facilities reported at least one antiretroviral or TB related medicine out of stock on the day of contact and $36 \%$ during the previous three months. ${ }^{48}$ These challenges have increased during the covid-19 pandemic, ${ }^{49}$ requiring emphasis on maintaining sexual and reproductive health services in high burden and fragile settings. ${ }^{50}$ Modelling illustrates that a six month disruption in prevention of motherto-child transmission services during the covid-19 lockdown for 50\% of people could result in 2.7 times more infants born with HIV in one year. ${ }^{51}$ Consequently, we need concerted efforts to monitor, achieve, and sustain undetectable viral loads throughout breastfeeding.

\section{Conclusion}

Antiretroviral treatment effectively reduces viral load (and mother-to-child transmission), but for a variety of reasons many women do not effectively achieve and maintain undetectable viral loads throughout pregnancy and breastfeeding. Current approaches to viral load monitoring are poorly implemented and the response to high viral load measurements is suboptimal; thus the risk of breast milk transmission by women taking antiretroviral drugs has not been eliminated. Studies reporting maternal plasma viral load and breast milk mother-to-child transmission, together or separately, are difficult to compare-viral load measurements occur at different time points and cut-offs for viral suppression and laboratory techniques differ. It is therefore important to rapidly scale-up other strategies such as frequent point-of-care viral load testing coupled with timely implementation of additional interventions to reduce breast milk mother-to-child transmission among women taking antiretroviral drugs. However, the optimal frequency of viral load monitoring during breastfeeding still needs investigation. Data show that viraemia occurs during breastfeeding, providing strong justification for investigating the utility of interventions such as long acting antiretrovirals, broadly neutralising antibodies, or vaccines despite maternal antiretroviral treatment to eliminate breast milk transmission.

Competing interests: All authors have read and understood BMJ policy on declaration of interests and have no interests to declare. The article was funded by the South African Medical Research Council. The content is the sole responsibility of the authors and does not necessarily represent the official views of the organisations and funders.

Contributors and sources: AEG has led national surveys to monitor effectiveness of approaches for prevention of transmission. AEG assisted by PVdP conceptualised the paper and contributed to its direction and finalisation; GG and FD provided guidance and senior level oversight. EJA. J-PM, N Ngandu, N Nagot, RK, DM, and WC provided critical comments that helped shape the paper. All authors contributed to all drafts and approved the final version. AEG and PVdP contributed equally.

Provenance and peer review: Commissioned; externally peer reviewed.

This article is part of a series proposed by the South African Medical Research Council and commissioned by The BMJ. The BM/ retained full editorial control over external peer review, editing, and publication. Open access fees are funded by SAMRC.

Ameena E Goga, professor ${ }^{1,2}$

Philippe Van de Perre, professor $^{3}$

Nobubelo Ngandu, researcher ${ }^{1}$

Nicolas Nagot, professor ${ }^{3}$

Elaine J Abrams, professor ${ }^{4}$

Dhayendre Moodley, senior research scientist; associate professor ${ }^{5,6}$

Rachel King, assistant professor ${ }^{3,7}$

Jean-Pierre Molès, researcher ${ }^{3}$

Witness Chirinda, researcher ${ }^{1}$

Gabriella Scarlatti, director $^{8}$

Thorkild Tylleskär, professor ${ }^{9}$

Gayle G Sherman, professor ${ }^{10,11}$

Yogan Pillay, country director; senior global director ${ }^{12}$

François Dabis, professor ${ }^{13,14}$

Glenda Gray, president ${ }^{1}$

${ }^{1}$ South African Medical Research Council, Pretoria and Cape Town, South Africa

${ }^{2}$ University of Pretoria, Pretoria, South Africa

${ }^{3}$ Pathogenesis and Control of Chronic and Emerging Infections, University of Montpellier, INSERM, Etablissement Français du Sang; CHU Montpellier, Montpellier, France

${ }^{4}$ ICAP at Columbia, Mailman School of Public Health, Columbia University, New York, USA

${ }^{5}$ Department of Obstetrics and Gynaecology, School of Clinical Medicine, University of KwaZulu Natal, Durban, South Africa

${ }^{6}$ Centre for AIDS Research in South Africa, Durban, South Africa

${ }^{7}$ UCSF, San Francisco, CA, USA

${ }^{8}$ Viral Evolution and Transmission Unit, Division of Immunology, Transplantation and Infectious Diseases, IRCCS Ospedale San Raffaele, Milan, Italy

${ }^{9}$ Centre for International Health, University of Bergen, Bergen, Norway

${ }^{10}$ Department of Paediatrics and Child Health, Faculty of Health Sciences, University of the Witwatersrand, Johannesburg, South Africa

${ }^{11}$ Centre for HIV \& STI, National Institute for Communicable Diseases, National Health Laboratory Services, Johannesburg, South Africa

${ }^{12}$ Clinton Health Access Initiative, South Africa

${ }^{13}$ Agence Nationale de Recherche sur le Sida et les Hépatites Virales (ANRS), Paris, France

${ }^{14}$ Université Bordeaux, ISPED, Centre INSERM U1219-

Bordeaux Population Health, Bordeaux, France
Correspondence to: AE Goga

Ameena.Goga@mrc.ac.za

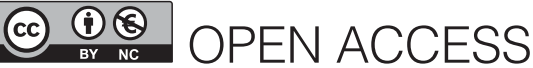

This is an Open Access article distributed in accordance with the Creative Commons Attribution Non Commercial (CC BY-NC 4.0) license, which permits others to distribute, remix, adapt, build upon this work non-commercially, and license their derivative works on different terms, provided the original work is properly cited and the use is non-commercial. See: http://creativecommons.org/licenses/by-nc/4.0/.

\section{Check for updates}

1 Van de Perre P, Rubbo P-A, Viljoen J, et al. HIV-1 reservoirs in breast milk and challenges to elimination of breast-feeding transmission of HIV-1. Sci Transl Med 2012;4:143sr3. doi:10.1126/ scitranslmed.3003327

2 Molès JP, Tuaillon E, Kankasa C, et al. Breastmilk cell trafficking induces microchimerism-mediated immune system maturation in the infant. Pediatr Allergy Immunol 2018;29:133-43. doi:10.1111/pai.12841

3 Pannaraj PS, Li F, Cerini C, et al. Association between breast milk bacterial communities and establishment and development of the infant gut microbiome. JAMA Pediatr 2017;171:647-54. doi:10.1001/ jamapediatrics.2017.0378

4 Liang G, Zhao C, Zhang H, et al. The stepwise assembly of the neonatal virome is modulated by breastfeeding. Nature 2020;581:470-4. doi:10.1038/s41586-020-2192-1

5 Zheng D, Liwinski T, Elinav E. Interaction between microbiota and immunity in health and disease. Cell Res 2020;30:492-506. doi:10.1038/s41422-0200332-7

6 Victora CG, Horta BL, Loret de Mola C, et al. Association between breastfeeding and intelligence, educational attainment, and income at 30 years of age: a prospective birth cohort study from Brazil. Lancet Glob Health 2015;3:e199-205. doi:10.1016/ S2214-109X(15)70002-1

7 National Department of Health SA. Protocol for providing a comprehensive package of care for the prevention of mother to child transmission of HIV (PMTCT) in South Africa. DH, 2001

8 World Health Organization. Prevention of HIV in infants and young children: review of evidence and WHO's activities. 2002. https://apps.who.int/iris/ handle/10665/67344

9 World Health Organization. Strategic approaches to the prevention of HIV infection in children: Report of WHO meeting, Morges Switzerland 20-22 Mar 2002. https://www.who.int/hiv/pub/mtct/pub35/en/

10 Mallampati D, MacLean RL, Shapiro R, et al. Optimal breastfeeding durations for HIV-exposed infants: the impact of maternal ART use, infant mortality and replacement feeding risk. J Int AIDS Soc 2018;21:e25107. doi:10.1002/jia2.25107

11 Jamieson DJ, Chasela CS, Hudgens MG, et al, BAN study team. Maternal and infant antiretroviral regimens to prevent postnatal HIV-1 transmission: 48-week follow-up of the BAN randomised controlled trial. Lancet 2012;379:2449-58. doi:10.1016/ S0140-6736(12)60321-3

12 Kumwenda NI, Hoover DR, Mofenson LM, et al. Extended antiretroviral prophylaxis to reduce breast-milk HIV-1 transmission. N Engl / Med 2008;359:119-29. doi:10.1056/NEJMoa0801941

13 Bedri A, Gudetta B, Isehak A, et al, Six Week Extended-Dose Nevirapine (SWEN) Study Team. Extended-dose nevirapine to 6 weeks of age for infants to prevent HIV transmission via breastfeeding in Ethiopia, India, and Uganda: an analysis of three randomised controlled trials. Lancet 2008;372:30013. doi:10.1016/S0140-6736(08)61114-9 
14 Nagot N, Kankasa C, Tumwine JK, et al, ANRS 12174 Trial Group. Extended pre-exposure prophylaxis with lopinavir-ritonavir versus lamivudine to prevent HIV-1 transmission through breastfeeding up to 50 weeks in infants in Africa (ANRS 12174): a randomised controlled trial. Lancet 2016;387:56673. doi:10.1016/S0140-6736(15)00984-8

15 World Health Organization. Use of antiretroviral drugs for treating pregnant women and preventing HIV infection in infants. 2012. https://www.who.int/hiv/ PMTCT_update.pdf.

16 Goga AE, Dinh T-H, Essajee S, et al. What will it take for the Global Plan priority countries in Sub-Saharan Africa to eliminate mother-to-child transmission of HIV?BMC Infect Dis 2019;19(suppl 1):783. doi:10.1186/s12879-019-4393-5

17 World Health Organization, Unicef. 2016 Guideline update on HIV and infant feeding. 2016. https://apps.who.int/iris/bitstream/hand le/10665/246260/9789241549707-eng.pdf;jsess ionid $=55$ E4F2B301A3270D6E75753733DFB899?s equence $=1$.

18 World Health Organization. Consolidated guidelines on the use of antiretroviral drugs for treating and preventing HIV infection 2016: recommendations for a public health approach. 2nd ed. 2016. https:// apps.who.int/iris/handle/10665/208825.

19 Van de Perre P, Goga A, Ngandu N, et al. Eliminating post-natal HIV transmission in high incidence areas: need for complementary biomedical interventions. ancet HIV [forthcoming].

20 Goga A, Singh Y, Jackson D, et al. Is elimination of vertical transmission of HIV in high prevalence settings achievable?BM/ 2019;364:1687 doi:10.1136/bmj.1687

21 Goga A, Singh Y, Jackson D, et al. How are countries in sub-Saharan African monitoring the impact of programmes to prevent vertical transmission of HIV?BM/ 2019:364:1660 doi:10.1136/bmi.1660

22 Giuliano M, Andreotti M, Liotta G, et al. Maternal antiretroviral therapy for the prevention of motherto-child transmission of HIV in Malawi: maternal and infant outcomes two years after delivery. PLoS One 2013;8:e68950. doi:10.1371/journal. pone.0068950

23 Davis NL, Miller WC, Hudgens MG, et al, BAN study team. Maternal and breastmilk viral load: impacts of adherence on peripartum HIV infections averted-the breastfeeding, antiretrovirals, and nutrition study. I Acquir Immune Defic Syndr 2016;73:572-80. doi:10.1097/QAl.0000000000001145

24 Shapiro RL, Hughes MD, Ogwu A, et al. Antiretroviral regimens in pregnancy and breast-feeding in Botswana. N Engl J Med 2010;362:2282-94. doi:10.1056/NEIMoa0907736

25 Waitt C, Low N, Van de Perre P, Lyons F, Loutfy M, Aebi-Popp K. Does U=U for breastfeeding mothers and infants? Breastfeeding by mothers on effective treatment for HIV infection in high-income settings. Lancet HIV 2018;5:e531-6. doi:10.1016/S23523018(18)30098-5

26 Myer L, Phillips TK, McIntyre JA, et al. HIV viraemia and mother-to-child transmission risk after antiretroviral therapy initiation in pregnancy in Cape Town, South Africa. HIV Med 2017;18:80-8. doi:10.1111/hiv.12397
27 World Health Organization. Global guidance on processes and criteria for validation: Elimination of mother-to-child transmission of HIV and syphilis. 2nd ed. Geneva, 2017. https://apps.who.int/iris/bitstream/ handle/10665/259517/9789241513272-eng. pdf?sequence $=1$

28 Bispo S, Chikhungu L, Rollins N, Siegfried N, Newell ML. Postnatal HIV transmission in breastfed infants of HIV-infected women on ART: a systematic review and meta-analysis. J Int AIDS Soc 2017;20:21251. doi:10.7448/IAS.20.1.21251

29 Landes M, van Lettow M, Nkhoma E, et al. Low detectable postpartum viral load is associated with HIV transmission in Malawi's prevention of mother-to-child transmission programme. J Int AIDS Soc 2019;22:e25290. doi:10.1002/jia2.25290

30 Hessell AJ, Jaworski JP, Epson E, et al. Early short-term treatment with neutralizing human monoclona antibodies halts SHIV infection in infant macaques. NatMed 2016:22:362-8. doi:10.1038/nm.4063

31 Shapiro MB, Cheever T, Malherbe DC, et al. Singledose bNAb cocktail or abbreviated ART postexposure regimens achieve tight SHIV control without adaptive immunity. Nat Commun 2020;11:70. doi:10.1038/s41467-019-13972-y

32 Chen YQ, Young A, Brown ER, et al. Population attributable fractions for late postnatal mother-tochild transmission of HIV-1 in Sub-Saharan Africa. J Acquir Immune Defic Syndr 2010;54:311-6. doi:10.1097/QAl.0b013e3181d61c2e

33 Lesosky M, Raboud JM, Glass T, et al. Comparison of guidelines for HIV viral load monitoring among pregnant and breastfeeding women in sub-Saharan Africa. AIDS 2020;34:311-5. doi:10.1097/ QAD.0000000000002400

34 Lesosky M, Glass T, Mukonda E, Hsiao N-Y, Abrams EJ, Myer L. Optimal timing of viral load monitoring during pregnancy to predict viraemia at delivery in HIV-infected women initiating ART in South Africa: simulation study. J Int AIDS Soc 2017;20(suppl 7). doi:10.1002/jia2.25000

35 Haas AD, Keiser O, Balestre E, et al, leDEA southern Africa, east Africa, and west Africa. Monitoring and switching of first-line antiretroviral therapy in adult treatment cohorts in sub-Saharan Africa: collaborative analysis. Lancet HIV 2015;2:e271-8. doi:10.1016/S2352-3018(15)00087-9

36 Global Network of People living with HIV. Report on stigma index survey. Thailand, 2010. https://www. aidsdatahub.org/sites/default/files/resource/stigmaindex-thailand-2009.pdf

37 National Department of Health. Guideline for the prevention of mother to child transmission of communicable infections. National Department of Health, 2019

38 Koss CA, Natureeba P, Kwarisiima D, et al. Viral suppression and retention in care up to 5 years after initiation of lifelong ART during pregnancy (Option $\mathrm{B}_{+}$) in rural Uganda. J Acquir Immune Defic Syndr 2017:74:279-84. doi:10.1097/ QAl.0000000000001228

39 Nichols BE, Girdwood SJ, Shibemba A, et al. Cost and impact of dried blood spot versus plasma separation card for scale-up of viral load testing in resourcelimited settings. Clin Infect Dis 2020;70:1014-20. doi:10.1093/cid/ciz338
40 Yotebieng M, Mpody C, Ravelomanana NL, et al, CQI-PMTCT study team. HIV viral suppression among pregnant and breastfeeding women in routine care in the Kinshasa province: a baseline evaluation of participants in CQI-PMTCT study. J Int AIDS Soc 2019;22:e25376. doi:10.1002/jia2.25376

41 Carmona S, Seiverth B, Magubane D, Hans L, Hoppler M. Separation of plasma from whole blood using the cobas plasma separation card: a compelling alternative to dried blood spots for the quantification of HIV-1 viral load. J Clin Microbiol 2019;57:e01336 18. doi:10.1128/JCM.01336-18

42 Drain PK, Dorward J, Violette LR, et al. Point-of-care HIV viral load testing combined with task shifting to improve treatment outcomes (STREAM): findings from an open-label, non-inferiority, randomised controlled trial. Lancet HIV 2020;7:e229-37. doi:10.1016/S2352-3018(19)30402-3

43 World Health Organization. Updated recommendations on HIV prevention, infant diagnosis, antiretroviral initiation and monitoring. WHO, 2021

44 Drain PK, Dorward J, Bender A, et al. Point-ofcare HIV viral load testing: an essential tool fo a sustainable global HIV/AIDS response. Clin Microbiol Rev 2019;32:e00097-18. doi:10.1128/ CMR.00097-18

45 Nicholas S, Poulet E, Wolters L, et al. Point-ofcare viral load monitoring: outcomes from a decentralized HIV programme in Malawi. J Int AIDS Soc 2019;22:e25387. doi:10.1002/jia2.25387

46 UNICEF DATA. Antenatal care: Maternal and newborn health coverage database April 2020. https://data. unicef.org/topic/maternal-health/antenatal-care/

47 World Health Organization. Global report on early warning indications of HIV drug resistance 2016. https://www.who.int/hiv/pub/drugresistance/ewihivdr-2016/en/

48 Hwang B, Shroufi A, Gils T, et al. Stock-outs of antiretroviral and tuberculosis medicines in South Africa: A national cross-sectional survey. PLoS One 2019;14:e0212405. doi:10.1371/journal. pone.0212405

49 Geach C. Lockdown putting a strain on SA's antiretrovirals stocks. Weekend Argus, 2020

50 Tran NT, Tappis H, Spilotros N, Krause S, Knaster S, Inter-Agency Working Group on Reproductive Health in Crises. Not a luxury: a call to maintain sexual and reproductive health in humanitarian and fragile settings during the COVID-19 pandemic. Lancet Glob Health 2020;8:e760-1. doi:10.1016/S2214 109X(20)30190-X

51 Jewell BL, Mudimu E, Stover J, et al, HIV Modelling Consortium. Potential effects of disruption to HIV programmes in sub-Saharan Africa caused by COVID-19: results from multiple mathematica models. Lancet HIV 2020;7:e629-40. doi:10.1016/ S2352-3018(20)30211-3

Supplementary tables summarising studies assessing risk of mother-to-child transmission and viral load in mothers taking antiretroviral drugs

Cite this as: BMJ 2021;374:n1697

http://dx.doi.org/10.1136/bmj.n1697 